\title{
Smartphone Sensor Accelerometer Data for Human Activity Recognition Using Spiking Neural Network
}

\author{
Nor Surayahani Suriani and Fadilla 'Atyka Nor Rashid
}

\begin{abstract}
Recognizing human actions is a challenging task and actively research in computer vision community. The task of human activity recognition has been widely used in various application such as human monitoring in a hospital or public spaces. This work applied open dataset of smartphones accelerometer data for various type of activities. The analogue input data is encoded into the spike trains using some form of a rate-based method. Spiking neural network is a simplified form of dynamic artificial network. Therefore, this network is expected to model and generate action potential from the leaky integrate-and-fire spike response model. The leaning rule is adaptive and efficient to present synapse exciting and inhibiting firing neuron. The result found that the proposed model presents the state-of-the-art performance at a low computational cost.
\end{abstract}

Index Terms-Activity recognition, spiking neural network, accelerometer sensor, spike train, firing rate

\section{INTRODUCTION}

Human activity recognition (HAR) is an important area of research in ubiquitous computing, human behaviour analysis and human-computer interaction. Research in these areas employ different machine learning algorithms to recognize simple and complex activities such as walking, running, cooking, etc. Particularly, recognition of daily activities is essential for maintaining healthy lifestyle, patient rehabilitation and activity shifts among the elderly citizens that can help to detect and diagnose serious illnesses. Therefore, human activity recognition framework provides mechanism to detect both postural and ambulatory activities, body movements and actions of users using different multimodal data generated by variety of sensors. Accelerometer sensor have been used in monitoring elderly, healthcare and rehabilitation systems to recognize human activities in mobile devices before smartphone available in the market. Nowadays smartphones became a must have gadget for our daily lives which we bring everywhere. People used smartphone at anytime and anywhere. Many smartphones are equipped with various sensors such as accelerometers, GPS, light sensors, gyroscope, temperature sensor, etc. These sensors have become a rich data source to develop real-time Android applications to measure various aspect of daily life. In terms of battery efficiency, the battery

Manuscript received July 12, 2019; revised April 7, 2020. This work was supported by Ministry of Higher Education (MOHE) under Fundamental Research Grant Scheme.

The authors are with the Universiti Tun Hussein Onn Malaysia, Malaysia (Corresponding author: Nor Surayahani Suriani; e-mail: nsuraya@uthm.edu.my, fadilla.atyka@gmail.com). can even last longer (more than 1 day). Even our smartphone can track what we do and also connect with other devices such as mobile cloud computing for other purposes.

Therefore, this research utilize smartphone data which have built-in sensor instead of collecting data from single accelerometer sensor in a normal system [1]. The advantage of using smartphone data are low installation cost, easy-to-use and slightly contains unobtrusive data. We chose open dataset from WISDM for training and testing purposes. This dataset contains typical activity like walking, sitting, etc.

In the state of the art, accelerometer sensor is the main sensor used for activity recognition. For example, accelerometer sensor has lots of potential in the field of rehabilitation and healthcare to emphasize the clinical applications of fall detection during rehabilitation treatment [2]. In classification phase for activity recognition system, the role of this sensor in performing overall recognition is evaluated using K-nearest neighbor classifier [3]. Author in [4], used multi-class Support Vector Machine (SVM) to classified accelerometer signal frequency signal pattern extracted from Fast Frequency Transform. To study the behavior of users, [5] implemented real-time activity recognition using hierarchical hidden Markov model.

This paper utilizes the extracted accelerometer sensor data collected using smartphone to recognize various types of human activities. This paper proposed spiking neural network (SNN) model to analyze the spike which highly informative for actions recognition. This paper is organized as follows: Section II briefly reviews related work in HAR using smartphone data. Sections III formulate the problems based feature extraction using SNN model. In Section IV, presents experimental results and discussions of the proposed approaches. Finally, conclusions are drawn in Section V.

\section{RELATED WORKS}

The research in HAR using smartphones accelerometer sensor has attract more practical applications especially in real-time classification of user activity. The challenge arises from existing works are on how to select acceptable time segments size within average interval duration of 2-10s. The experiments were designed and controlled under certain types of dataset. Results in [6] shows quite good performance with smaller time interval.

Other than that, selection of methods for feature extraction also play an important role in the performance of HAR. Existing research tends towards adapting deep learning or convolutional neural network (CNN) [7] in HAR. Normally, the raw input for this kind of machine learning are images. Majority of research are concerned with the idea of 
representation learning which focusing on how best to represent the raw signal data using deep leaning or CNN. For example, EEG signal data [8] is normalized at zero mean and standard deviation before feed into the $\mathrm{CNN}$ for training and testing. The CNN is implemented because it has both shift and translational invariance. In [9] and [10] image as an input to the CNN, which was similar to how an RGB data was processed by CNN. While [11] applied Discrete Fourier Transform to sensor image and extract features for further classification. Author in [12] also applied CNN for human activity recognition to detect any abnormalities occurred by estimated scale and velocity for different types of activities using Histogram of Oriented Optical Flow (HOOF) technique.

Study in SNN as the powerful neural network approaches has motivated researcher to focus on bioinspired methods for pattern recognition [13], [14]. SNN also learn to convert speech signal into spike train signatures which are distinguishable for other speech signals to represent different words [15]. In [16], SNN model is used to model balance brain interface using EEG pattern data. Author in [17] applied SNN model to recognizes different types walking pattern. The evaluation of SNN model also able to classify motor imagery EEG signals [18] due to robustness in pattern recognition compared to other classifiers like Linear Discriminant, Feed Forward Neural Network and Radial Basis Function. Further optimization technique applies for weights and delay settings. The acceleration data for each axis $x, y$ and $z$ are calculated by the integration of velocities. Fuzzy spiking neuron model is applied to observe and understand human behaviors in development of humanoid robot [19]. The person is extracted from measured data by the sensor network.

Inspired by biological human perception, accelerometer sensor data is encoded and model to match with spiking activity of cells. Then the extracted nonlinear frequency information from the spike trains is then classified using SVM for real-time surface texture classification in neurorobotic research [20]. Again, in wildlife behavior monitoring research, SNN model is implemented to analyze the data obtained from the GPS sensor to track animal positions and the behavior [21].

Over the last century, the first generation of neural network started with perceptron until today the deep learning networks trained using gradient descent. This $3^{\text {rd }}$ generation of neural network believes that SNN will be the next generation of machine learning which compute biological-realistic models of human brain.

In this research, SNN model is employed to encoded motion pattern of rehabilitation monitoring system using 3D Gabor spatio-temporal filter in V1 and MT layer to form active motion map according to direction, speed and orientation of object movement based on Gaussian distribution [22]. SNN model are inspired by the brain and communication of neurons which useful for information transformation via action potentials (spikes) through adaptive synapses. Spiking neuron is generated when the average of changes in membrane potential exceed a spiking threshold, resulting from stimulation. The rate of spike generation and the temporal pattern of spike train carry significant information of the stimuli. The following section will explain the details of SNN architectures.

\section{SPIKING NeURON NeTwORK MODEL}

SNN model incorporating spatial-temporal information in communication and computation, like real neurons. The neuronal signals consist of short electrical pulses which known as action potentials or spikes. Spike is the incoming signals that alter the voltage of the neuron and when this reaches above a threshold-value the neuron sends out an action potential itself. While the chain of action potentials emitted by a single neuron is called spike train. These individual spikes are sparse in time, so each spike has high information content. The knowledge representation in time and space makes SNNs unique to perform brain-like computations and to understand the brain data/activity in a spatio-temporal pattern.

Considering an accelerometer dataset in $M \times N \times T$ matrix, where $\mathrm{M}$ indicates the total number of data, $\mathrm{N}$ is the $x, y, z$ axis of accelerometer sensor and $T$ is total number of trials. Fig. 1 shows the general stages of classification using SNN model. The accelerometer data act as the raw input data, preprocess signal data is transformed into spike train input dataset. The spike train data work as the initial input of the SNN model. Further setting weight parameter and optimization of the SNN is done within the network. Finally, the similarity score of the network output determine type of the output class.

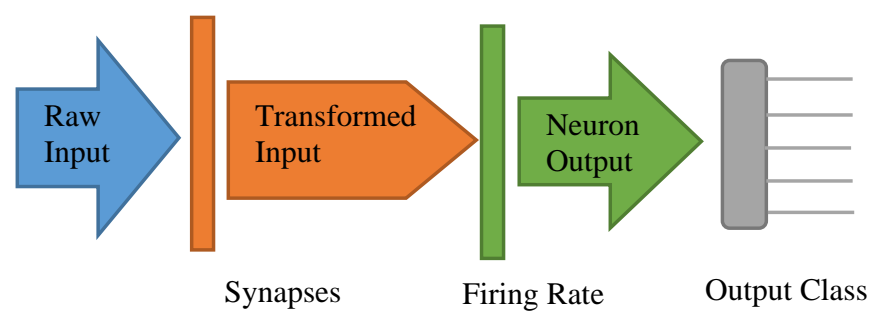

Fig. 1. Stages for SNN model.

For firing rate and spike generation, there are many different schemes for the use of spike timing information in neural computation. The most widely used and best-known model of threshold fire neurons, and spiking neurons in general, is the Integrate-and-Fire neuron. The Integrate-and-Fire neuron is the simplest spiking neuron models and easy to analyzed and simulated. The neuron receives all the weighted spikes from the presynaptic neurons via several synapses. The neuron generates spike trains according to a conductance-driven Integrate-and-Fire equation as follows:

$$
\begin{aligned}
& \frac{d V(t)}{d t}=G_{\theta}^{e x c}(x, y, t)\left(E^{e x c}-V(t)\right) \\
& +G_{\theta}^{i n h}(x, y, t)\left(E^{i n h}-V(t)\right)-g^{L} V(t)
\end{aligned}
$$

where

$G_{i}^{\text {exc }}(t)=$ excitatory conductance

$G_{i}^{i n h}(t)=$ inhibitory conductance

$E^{L}=$ resting potential 
$E^{e x c}=$ membrane cell potential

$g^{L}=$ inert leaks in the cells's membrane

The spiking neuron models initial voltage, threshold voltage and reset voltage of spiking neural model are set to $80 \mathrm{mv},-10 \mathrm{mv}$ and $0 \mathrm{mv}$ respectively. Spike when $V=1$, otherwise reset. The excitatory conductance $G_{i}^{\text {exc }}(t)$ for $(x, y)$ in the receptive field $V 1$ cells are given as

$$
G_{i}^{e x c}(x, y, t)=k_{c} C_{\theta}(x, y, t)
$$

where $k_{c}$ is amplification factor. When cell fires a spike, the inhibitory conductance, $G_{i}^{i n h}(t)$ is generated in the neighborhood cell which has similar direction, orientation angle and speed. The inhibitory conductance, $G_{i}^{i n h}(t)$ given as

$$
G^{i n h}=G_{\max }^{i n h} e^{-\frac{d^{4}}{2 R_{i n h}^{2}}}
$$

where $d$ is the Euclidean distance between each cell with the neighborhood cell and $R_{i n h}$ is the size of receptive field. In order to achieve the input patterns, which will indicate the same class of activity, the generated cell almost have the same firing rate. While different classes will generate different firing rates with the average spiking rate of each class is widely separated. This is enough to discriminate among different types of HAR.

The firing rate is calculated by the summation of spikes that have been fired by neuron, $i$ as shown in the firing rate equation as follow:

$$
\gamma_{i}(t, \Delta t)=\frac{\eta_{i}\left(t-\Delta_{t} t\right)}{\Delta_{t}}
$$

The general learning rule for the synapses is given by:

$$
\Delta w=\left\{\begin{array}{l}
A^{+\frac{\Delta t}{e^{r+}}} \text { if } \Delta t<0 \\
A^{-\frac{-\Delta t}{e^{r-}}} \text { if } \Delta t>0
\end{array}\right.
$$

where, $\Delta t=t_{\text {pre }}-t_{\text {post. }}$. Here the goal is that weights are changed such that the next (after this training iteration) postsynaptic spike occurs closer to the pre-synaptic spike. The weights are then changed according to the relaxation rule:

$$
w_{\text {new }}=\left\{\begin{array}{l}
w_{\text {old }}+\eta \Delta w\left(w_{\text {max }}-w_{\text {old }}\right) \text { if } \Delta w \geq 0 \\
w_{\text {old }}+\eta \Delta w\left(w_{\text {old }}-w_{\text {min }}\right) \text { if } \Delta w<0
\end{array}\right.
$$

Here, $\eta$ is the learning rate. For excitatory synapses $w_{\min }=$ 0 and $w_{\max }=1$, whereas for inhibitory synapses $w_{\min }=-1$ and $w_{\max }=0$.

In classification part, the predefine spike sequences for different input patterns and the learning neuron is trained to fire a desired sequence of spikes when a corresponding pattern is present. Euclidean distance measure of spike timing was adopted to measure the similar degree between the target and observed output spike trains. When the actual output spike train is the same with the desired output spike train, the measure $C=1$, and the $C$ decreases towards 0 for loosely similarity pattern of spikes.

\section{RESUlts AND ANALYSIS}

\section{A. Dataset}

We present a comparative performance evaluation SNN model for WISDM dataset [23]. The dataset is made by six different activities, collected under controlled laboratory environment. The activities are walking, jogging, going upstairs and downstairs, sitting and standing position. WISDM data is collected at $20 \mathrm{~Hz}$ from both Android phone and smartwatch. The dataset has been partitioned into 30 frames per sample and preprocess to remove the unnecessary noisy signal before being generated as a spike train.

\section{B. Experimental Setting}

After few learning epochs, the neuron in training set can generate desired output spike trains for every class of activity input pattern. The WISDM dataset divided into 10-fold cross validation. The performance of HAR was primarily evaluated based on the percentage accuracy of activity recognized correctly. The accuracy for each iteration is computed by comparing the equivalence of the predicted activity class and ground truth label of the activity label for each feature vector and illustrated in confusion matrix.

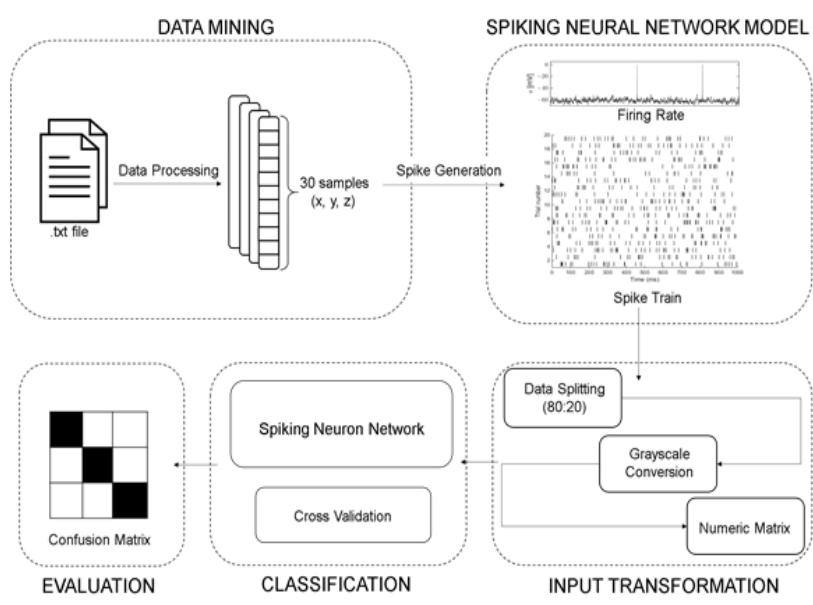

Fig. 2. General Framework for SNN Model performance evaluation.

Fig. 2 emphasized the general framework for SNN Model performance evaluation. WISDM dataset has been partitioned into 30 frames per sample before being generated as a spike train. Spike trains then being split into training and test data with the ratio of 80-20 respectively. Next, the spike train is taken as an input and converted into gray scale images. The converted images being transformed into numeric matrix for training in classification phase. Scores for each data are then obtained from the network output. Finally, we illustrated the accuracy by performing confusion matrix in the evaluation phase.

\section{SNN Analysis}

We verify the variability of results for this dataset using the proposed SNN model. The result indicate that the information extracted from the input patterns encode neuron 
and generate unique spikes for each class of activity. The uniqueness of each spikes mean firing rate for different types of activities justify the relevant responses cells for action task purposes.

The performance accuracy depends on the selected window length of the size interval and sampling rate. The following classification results were carried out for selected size interval, which is 30 samples per second. This size interval was selected due to the acceptable or normal size interval used in previous work.

Based on the result, the accelerometer produces reasonable performance result using the proposed SNN model. These results can be verified for more extensive dataset of activities and using different size interval. Overall, the experiment was carried out using normal CPU and the computational time is only $2 \mathrm{~s}$ per 30 sample. The prediction is updated every $3 \mathrm{~s}$. This would be acceptable for real-time activity recognition. The neuron parameters used here are as follows: $\tau=80 \mathrm{~ms}, \theta$ $=4$ and $R_{\text {inh }}=1$ while the parameters $\gamma$ in the population encoding is 1.5. In this experiment, the SNN model has one hidden layer with 10 hidden neurons and only one output layer with one output neuron. There is no synapse between input neuron and hidden neuron but added 5 synapses between hidden neuron and output neuron.

\section{Spiking Train}

Fig. 3 and Fig. 4 show the sample of an average firing rate for 'walking' and 'sitting' activity. By looking at the x-axis, the activity can be classified based on the spike pattern generated along $0^{\circ}$ to $360^{\circ}$ orientation direction. Generic walking pattern generated unique spikes train for walking pattern distributed along the directions. While for sitting activity, sudden changes of spikes occur which indicate the change of movement from standing to sitting position. The range of firing rates for sitting activity is below $1000 \mathrm{~Hz}$ compared with walking activity fall within $1000-15000 \mathrm{~Hz}$. This indicate that the hit of action potential towards sitting activity is slower than normal walking activity.

Fig. 5 and Fig. 6 differentiate the firing rate between activity for going upstairs and downstairs. Sudden drop occur at the earlier stage, while repeated pattern firing up and down throughout the movement. This indicate that motion patterns of individual activities are significantly meaningful and firing rate data can carry meaningful information of the spatio-temporal features detection for different activities.

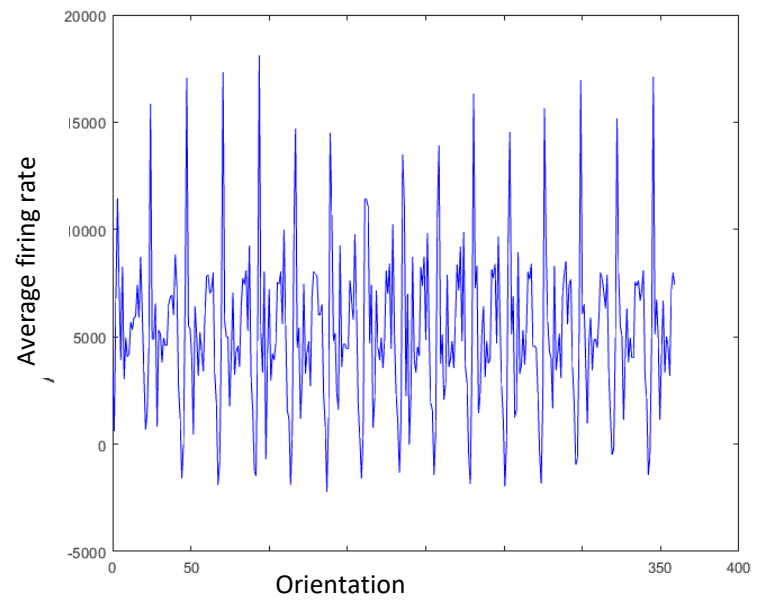

Fig. 3. The average firing rate for "walking' activity.

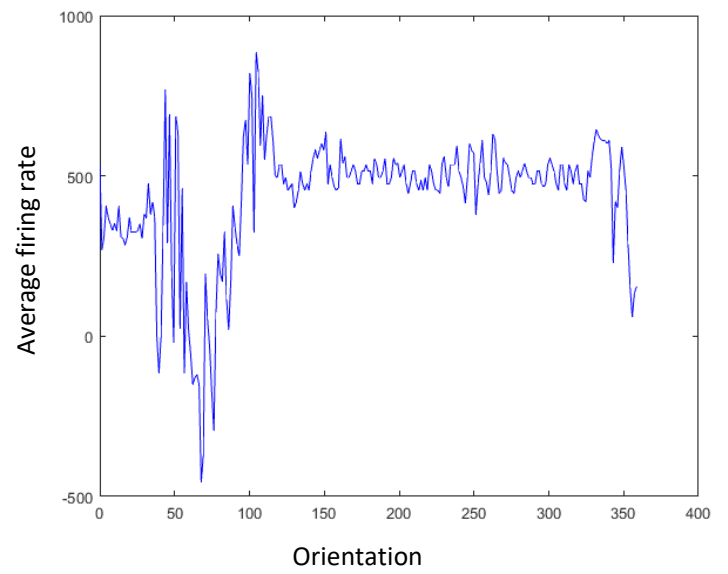

Fig. 4. The average firing rate for "sitting' activity.

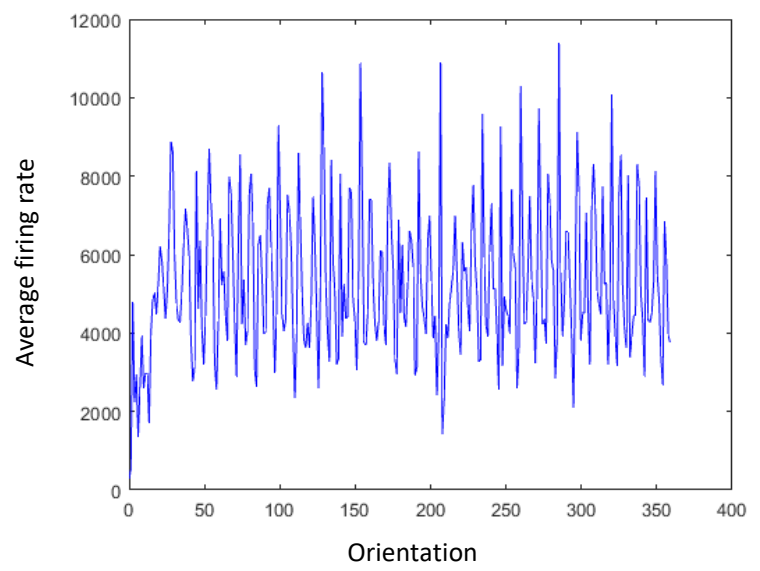

Fig. 5. The average firing rate for "going upstairs' activity.

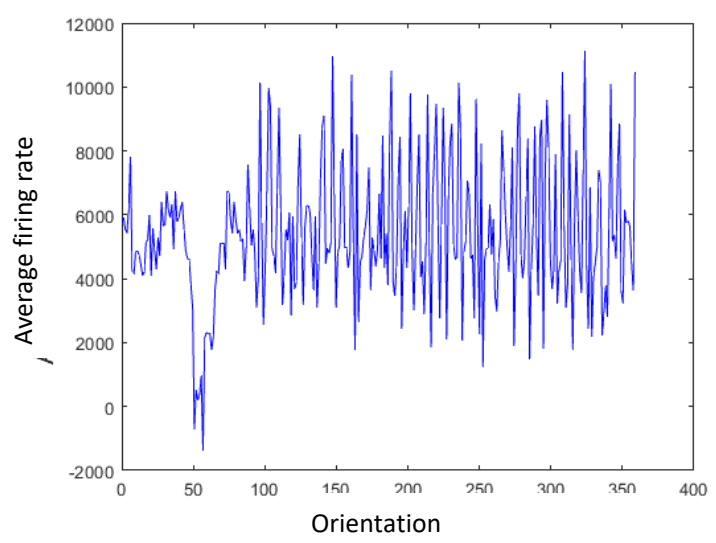

Fig. 6. The average firing rate for 'going downstairs' activity.

The series of firing rates encode the captured motion into spike trains. The spike trains capture motion of all accelerations within time interval at each frame. Fig. 7 and Fig. 8 show the example of spike trains also for walking and sitting activity. The figures depict that the pattern of the spikes is vary from one to another. Walking activity's spike patterns a bit scattered as the movement of the activity is non-static in contrast with sitting activity, the patterns more organized and structured as sitting activity involved in a static placement.

At this point, the number of synapse between neurons is selected carefully in order to prevent reduction of convergence rates and classification accuracy. While, if more synapses is added into the network, the learning efficiency will be affected with no improvement in convergence or 
classification accuracy.

In this paper, we employ spike train as the input to be transformed before being applied to Euclidean distance measure. As a result, Fig. 9 summarize the accuracy of SNN Model performance evaluation. The average of the accuracy is almost achieved $100 \%$ which describes each of the activity has their own uniquely spike patterns. Some information might get lost for certain activity, which may lead to a lower recognition rate. On the other hand, the SNN model capture spatio-temporal features naturally from the spike train analysis and able to classify the output class directly. There is no preprocessing needed to extract temporal features from the raw accelerometer dataset.

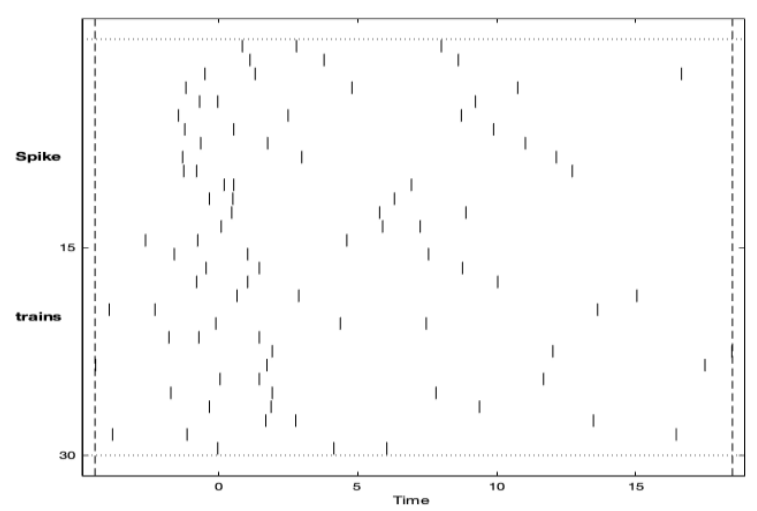

Fig. 7. The spike train for "walking' activity.

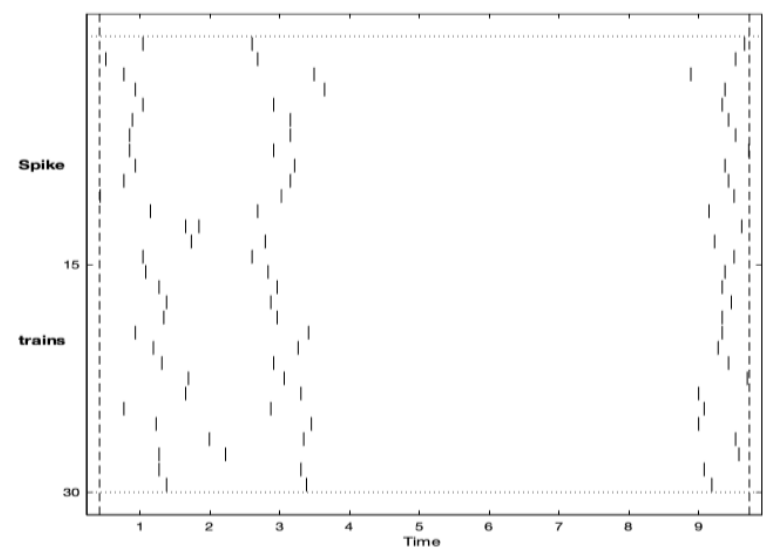

Fig. 8. The spike train for "sitting' activity.

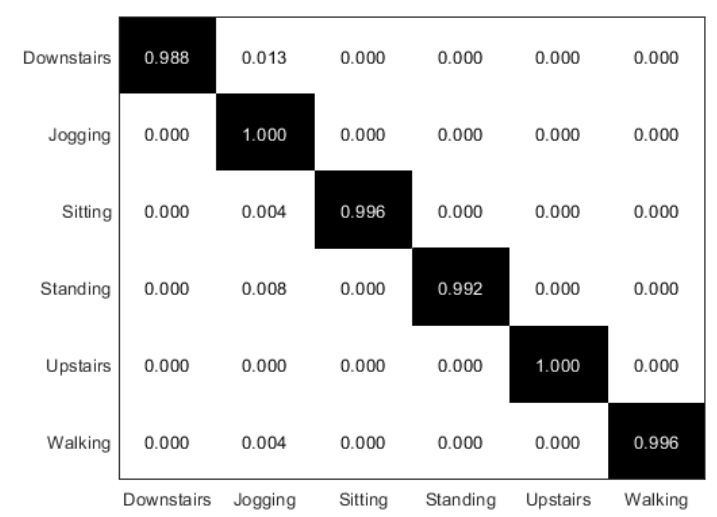

Fig. 9. Accuracy of SNN Model performance evaluation for 6 activities.

\section{CONCLUSION}

In this paper a new method to apply a spiking neuron in a pattern recognition task was proposed. This method is based on the firing rates produced with integrate-and-fire neuron when is stimulated. The proposed architecture cost at low computational time which can also run on mobile devices in real time. The evaluation performance result will be tested for another smartphone dataset collected under uncontrolled environment. Hence, further filtering at preprocessing process is needed to validate the proposed framework. Extensive dataset will be carried out for different user with different accelerometer devices calibrations.

Although the proposed SNN model for activity recognition is shown to be promising, few parameters in the network are determined heuristically. Hence, the final output is slightly depends on the dataset and parameter setting within the network after a few trials. Therefore, the proposed SNN framework need to be verified on more data sets. Further powerful search and optimization methods can be apply to tune the parameters of SNN model automatically to improve its performance. In conclusion, the initial results tested on popular dataset WISDM achieve our expectation. More comparison with state-of-the art approaches is needed in future work to evaluate the robustness of the proposed model.

\section{CONFLICT OF INTEREST}

The authors declare no conflict of interest.

\section{AUTHOR CONTRIBUTIONS}

N. S. Suriani wrote and finalized the whole paper, conducted the research in bio-inspired visual cortex system and this paper present some of the experimental results. While, Fadilla Atyka N. R. help in analyzed the data and wrote the paper for results and analysis part. All authors had approved the final version.

\section{ACKNOWLEDGMENT}

The author would like to express the deepest appreciation to the Ministry of Higher Education (MOHE) for funding this project through the Fundamental Research Grant Scheme (FRGS) grant of (No. 1584).

\section{REFERENCES}

[1] K. Kuspa and T. Pratkanis. (2013). Classification of Mobile Device Accelerometer Data for Unique Activity Identification. [Online]. Available:

http://cs229.stanford.edu/proj2013/PratkanisKuspa-ClassificationOfM obileDeviceAccelerometerDataforUniqueActivityIdentification.pdf

[2] N. S. Suriani, A. N. Fadilla, and N. Y. Yunos, "Optimal accelerometer placement for fall detection of rehabilitation patients," Journal of Telecommunication, Electronics and Computer Engineering, vol. 7, no. 25, 2018.

[3] C. P. Wu, W. S. Dasgupta, E. R. Ernesto and J. N. Gregnory, "Classification accuracies of physical activities using smartphone motion sensors," J. Med. Internet Res., vol. 14, no. 5, 2012.

[4] D. Anguita, A. Ghio, L. Oneto, X. Parra, and L. Reyes-Ortiz, "Human activity recognition on smartphones using a multiclass hardware-friendly support vector machine," in Proc. International Workshop on Ambient Assisted Living, 2012, pp. 216-223.

[5] Y.-S. Lee and S.-B. Cho, "Activity recognition using hierarchical hidden markov models on a smartphone with $3 \mathrm{~d}$ accelerometer," in Proc. International Conference on Hybrid Artificial Intelligence Systems, 2011, pp. 460-467.

[6] M. Kose, O. Incel, and C. Ersoy, "Online human activity recognition on smart phones," in Proc. Workshop on Mobile Sensing: From Smartphones and Wearables to Big Data, 2012.

[7] Henry, Teh, M. A. Al-garadia, and Uzoma., "Deep learning algorithms for human activity recognition using mobile and wearable sensor 
networks: State of the art and research challenges," Expert Syst. Appl., vol. 105, no. 1, pp. 233-261, 2018.

[8] U. R. Acharya, S. L. Oh, Y. Agiwara, J. H. Tan, and H. Adeli, "Deep convolutional neural network for the automated detection and diagnosis of seizure using EEG signals," Comput Biol Med., 2017.

[9] J. B. Yang, M. N. Nguyen, P. P. San, X. L. Li, and S. Krishnaswany, "Deep convolutional neural networks on multichannel time series for human activity recognition," in Proc. the 24th International Conference on Artificial Intelligence, 2015, p. 3995.

[10] F. Ordonez and D. Roggen "Deep convolutional and LSTM recurrent neural networks for multimodal wearable activity recognition," Sensors, vol. 16, no. 1, p. 115, 2016.

[11] W. Jiang and Z. Yin, "Human activity recognition using wearable sensors by deep convolutional neural networks," in Proc. the 23rd ACM International Conference on Multimedia, 2015, pp. 1307-1310.

[12] N. S. Suriani, A. N. Fadilla, M. H. Badrul, "Semantic object detection for human activity monitoring system," Journal of Telecommunication, Electronics and Computer Engineering, vol. 7, p. 115, 2018.

[13] R. A. V'azquez, "Pattern recognition using spiking neurons and firing rates," in Proc. Conference on Advances in Artificial Intelligence, 2010.

[14] P. Bawane, S. Gadariye, S. Chaturvedi, and A. A. Khurshid, "Object and character recognition using spiking neural network," in Proc. Materials Today, 2018, vol. 5, no. 1, pp. 360-366.

[15] A. Tavanaei and A. S. Maida, "A spiking network that learns to extract spike signatures from speech signals," Neurocomputing, vol. 240, pp. 191-199, 2017.

[16] R. Salazar-Varas and R. A. Vazquez, "Evaluating spiking neural models in the classification of motor imagery EEG signals using shor calibration sessions," Appl. Soft Comput. J., vol. 67, pp. 232-244, 2018.

[17] K. F. Mares, R. Baltazar, Á. C. Miguel, V. Zamudio, and L. Lemus, "A proposal to classify ways of walking patterns using spiking neura networks," Fuzzy Log. Augment. Neural Optim. Algorithms Theor. Asp. Real Appl., pp. 89-98, 2018.

[18] R.Salazar-Varasa and R. A.Vazquez, "Evaluating spiking neural models in the classification of motor imagery EEG signals using short calibration sessions," Appl. Soft Comput., vol. 67, pp. 232-244, 2018.

[19] N. Kubota, D. Tang, T. Obo, and S. Wakisaka, "Localization of human based on fuzzy spiking neural network in informationally structured space," in Proc. International Conference on Fuzzy Systems, 2010

[20] K. E. Friedl, A. R. Voelker, A. Peer, and C. Eliasmith, "Human-inspired neurorobotic system for classifying surface textures by touch," IEEE Robot. Autom. Lett., 2015.
[21] A. Linares-Barranco, A, Rios-Navarro, J. P. Dominguez-Morales, R Tapiador-Morales, M. Dominguez-Morales, and A Jimenez-Fernandez, "A sensor fusion horse gait classification by a spiking neural network on SpiNNaker," in Proc. International Conference on Artificial Neural Networks, 2016, pp. 36-44.

[22] N.S. Suriani, "Fall detection using visual cortex bio-inspired model for home-based physiotherapy system," Advances in Machine Learning And Signal Processing, Springer, vol. 6, no. 47, 2016.

[23] J. R. Kwapisz, G. M. Weiss, and S. A. Moore, "Activity recognition using cell phone accelerometers," in Proc. the Fourth International Workshop on Knowledge Discovery from Sensor Data, 2010.

Copyright $(9) 2021$ by the authors. This is an open access article distributed under the Creative Commons Attribution License which permits unrestricted use, distribution, and reproduction in any medium, provided the original work is properly cited (CC BY 4.0).

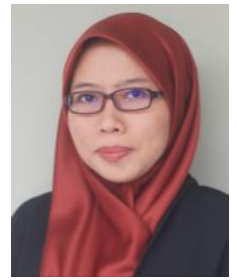

Nor Surayahani Suriani is a senior lecturer in Universiti Tun Hussein Onn Malaysia (UTHM). She received her $\mathrm{PhD}$ in electronics and computer system from Universiti Kebangasaan Malaysia (UKM) in 2015, and finished her master (Universiti Teknologi Malaysia) and Bac. Eng. (Universiti Putra Malaysia) in electronics and communication in 2007 and 2003, respectively. Her research interest focuses on computer vision, image processing and bioinspired visual cortex algorithm development. She has published in Q1 impact factor and Scopus journals mainly in image processing and bio-inspired computer vision areas especially in healthcare and activity monitoring applications.

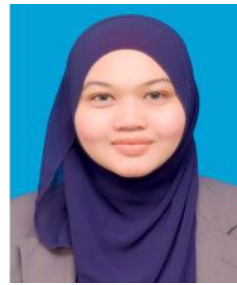

Fadilla A. Nor Rashid was born in Johor, Malaysia in 1991. She is a degree holder of computer network and security from Universiti Teknologi Malaysia and a masters degree of telecommunication engineering from Universiti Teknologi Mara. She is currently a $\mathrm{PhD}$ students in Computer Engineering Department at Universiti Tun Hussein Onn Malaysia. Her area of interests and research are in the computer vision, machine learning, and artificial intelligence. She has presented papers in conferences and published a paper in Scopus journals. 